Chirurg 2014 $\cdot 85: 450$

DOI 10.1007/s00104-014-2750-x

Online publiziert: 4. April 2014

(c) Springer-Verlag Berlin Heidelberg 2014

J. Reibetanz • C.-T. Germer

Klinik für Allgemein-, Viszeral-, Gefäß- und Kinderchirurgie, Universitätsklinik Würzburg

\title{
Ist die routinemäßige Koloskopie nach einer akuten Sigmadivertikulitis sinnvoll?
}

skopie, CT, Kontrastdarstellung) erfolgt war.

\section{Ergebnisse}

Systematic review and meta-analysis of the role of routine colonic evaluation after radiologically confirmed acute diverticulitis. Ann Surg 259:263-272

\section{Hintergrund}

In den letzten Jahren zeichnet sich ein Trend hin zum konservativen Management der akuten Divertikulitis ab. Zum Ausschluss von Malignität empfehlen internationale Leitlinien die Durchführung einer endoskopischen Abklärung des Kolons im Intervall nach akuter Divertikulitis. Diese Empfehlungen stammen jedoch aus einer Ära vor routinemäßigem Einsatz der Computertomographie (CT) in der Akutdiagnostik der Erkrankung. Die als notwendig erachtete "Intervallkoloskopie“ könnte daher eher die Schwächen der damaligen diagnostischen Methoden widerspiegeln als eine echte Indikation zum Ausschluss maligner Befunde im Kolon.

\section{Fragestellung und Methode}

Ziel dieser systematischen Literaturrecherche und Metaanalyse war die Überprüfung der Sinnhaftigkeit einer routinemäßigen Koloskopie nach einer Episode einer akuten Divertikulitis zum Ausschluss (prä-)neoplastischer Zweitbefunde im Kolon. Hierfür wurden aus den bekannten medizinischen Datenbanken alle relevanten Originalpublikationen extrahiert, bei denen eine Abklärung des Kolons (Koloskopie oder CT-Kolonographie) im Anschluss an eine bestätigte Episode einer akuten Divertikulitis (Endo-
Insgesamt 11 Studien mit 3358 Patienten wurden in die Metaanalyse eingeschlossen, wovon 1970 Patienten eine routinemäßige Abklärung des Kolons erhalten hatten. In der Mehrzahl der Studien erfolgte die Koloskopie innerhalb von 6 bis 8 Wochen nach dem Ereignis. Hierbei zeigte sich bei insgesamt 22 der 1970 untersuchten Patienten ein Malignom im Kolon, entsprechend einem gepoolten Risiko von 1,6\% (95\%-Konfidenzintervall (KI): 0,9-2,8\%). Wurden die Patienten nach dem Schweregrad der Divertikulitis stratifiziert, fand sich ein Malignom bei 5 von 1497 Patienten (8 Studien) mit unkomplizierter Divertikulitis (gepooltes Risiko: 0,7\%, 95\%-KI: $0,3-1,4 \%)$ vs. 6 von $79 \mathrm{~Pa}-$ tienten (5 Studien) mit komplizierter Divertikulitis (gepooltes Risiko: 10,8\%, 95\%KI: 5,2-21\%). Insgesamt 7 Studien berichteten zudem über die Häufigkeit prämaligner Polypen im Rahmen der Intervallkoloskopie und fanden ein gepooltes Risiko von $16,5 \%$ (95\%-KI: 11,2-23,8\%) für derartige Läsionen.

\section{Diskussion und Fazit des Reviewers}

Die aktuelle Literaturübersicht zeigt, dass die Wahrscheinlichkeit, durch eine Routinekoloskopie nach einem akuten Schub einer Sigmadivertikulitis ein Malignom zu detektieren mit 1,6\% vergleichsweise niedrig ist. Diese Einschätzung gilt jedoch nicht für komplizierte Stadien der Divertikulitis (Risiko eines malignen Befundes im Restkolon bis ca. $11 \%)$, sodass diese Patienten unbedingt einer Intervallkoloskopie zugeführt werden sollten! Im Gegensatz dazu scheinen maligne Befunde im Kolon bei Patienten nach unkomplizierter Sigmadivertikulitis mit $\mathbf{0 , 7 \%}$ sehr selten (und nicht häufiger als bei Screening-Koloskopien der asymptomatischen Bevölkerung [1]), sodass eine routinemäßige Koloskopie in diesen Fällen entbehrlich erscheint. Die vorliegende Arbeit plädiert daher für eine selektive Empfehlung zur Koloskopie in Abhängigkeit der Erkrankungsschwere der Divertikulitis. Da jedoch häufig Patienten betroffen sind, für die sich ohnehin aufgrund des Alters eine Vorsorgekoloskopie empfiehlt, kann man ein (konservativ therapiertes) Ereignis einer akuten Sigmadivertikulitis immer gut zum Anlass nehmen, die „Volkskrankheit kolorektales Karzinom“ gegenüber dem Patienten zu thematisieren und an die leitliniengerechte Vorsorge zu erinnern.

\section{Korrespondenzadresse}

Prof. Dr. C.-T. Germer

Klinik für Allgemein-, Viszeral-, Gefäß- und Kinderchirurgie, Universitätsklinik Würzburg, Oberdürrbacher Str. 6, 97080 Würzburg germer_c@klinik.uni-wuerzburg.de

Interessenkonflikt. J. Reibetanz und C.-T.Germer geben an, dass kein Interessenkonflikt besteht.

\section{Literatur}

1. Niv Y, Hazazi R, Levi Z, Fraser G (2008) Screening colonoscopy for colorectal cancer in asymptomatic people: a meta-analysis. Dig Dis Sci 53:3049-3054 